\title{
The role of cognitive inhibition in different components of arithmetic
}

\author{
Camilla Gilmore $\cdot$ Sarah Keeble $\cdot$ Sophie Richardson $\cdot$ \\ Lucy Cragg
}

Accepted: 13 December 2014 / Published online: 10 January 2015

(C) The Author(s) 2015. This article is published with open access at Springerlink.com

\begin{abstract}
Research has established that executive functions, the skills required to monitor and control thought and action, are related to achievement in mathematics. Until recently research has focused on working memory, but studies are beginning to show that inhibition skills-the ability to suppress distracting information and unwanted responses-are also important for mathematics. However, these studies employed general mathematics tests and therefore are unable to pinpoint how inhibition skills relate to specific components of mathematics. We explored how inhibition skills are related to overall achievement as well as factual, procedural and conceptual knowledge in 209 participants aged 11-12, 13-14 and adults. General mathematics achievement was more strongly related to inhibition measured in numerical compared with non-numerical contexts. Inhibition skills were related to conceptual knowledge in older participants, but procedural skills in younger participants. These differing relationships can shed light on the mechanisms by which inhibition is involved in mathematics.
\end{abstract}

\section{Introduction}

It is well established that learning mathematics presents a challenge for many children and young people. This can lead to negative consequences for job prospects and quality

\section{Gilmore $(\bowtie) \cdot$ S. Keeble}

Mathematics Education Centre, Loughborough University, Loughborough, Leicestershire LE11 3TU, UK

e-mail: c.gilmore@lboro.ac.uk

S. Richardson $\cdot$ L. Cragg

School of Psychology, University of Nottingham, Nottingham, UK of life (Parsons and Bynner 2005). Despite educational initiatives to improve mathematics achievement there has been a disappointing lack of improvement in mathematics outcomes in many Western societies (Vorderman et al. 2011). Given this lack of progress, researchers have attempted to better understand the cognitive processes that underlie mathematics performance. An improved theoretical understanding of the factors involved in mathematics processing can provide the starting point from which to develop pedagogy to support mathematics learning in all young people.

Over the past few decades, researchers have identified two classes of cognitive skills that are important for mathematics achievement. The first of these concerns domainspecific skills such as symbol knowledge, counting skill, and underlying numerical representations. Alongside these, researchers have identified domain-general skills which are involved in learning in many areas but which are particularly important for mathematics (e.g. language, IQ and spatial ability). Particular attention has been paid to executive functions-the skills required to monitor and control thought and action - and the role they play in learning and performing mathematics (see reviews by Cragg and Gilmore 2014; Bull and Lee 2014). Three types of executive functions have been identified: monitoring and manipulating information in mind (working memory), suppressing distracting information and unwanted responses (inhibition), and flexible thinking (shifting). To date, few models of mathematical cognition have considered the role of executive function skills, particularly inhibition. LeFevre et al. (2010) identified a role for attentional processes in their Pathways Model. This model proposed that attentional skills have a direct impact on mathematical performance in a variety of domains independent of linguistic or quantitative skills. However, the specific role of inhibition skill was not specified in this model. 
There is a wealth of evidence that working memory is involved in mathematics (see reviews by DeStefano and LeFevre 2004; Raghubar et al. 2010). More recently, the focus has turned to inhibition and it has been hypothesized that individuals with higher levels of inhibitory control are more successful in mathematics. In the sections below, we first review the existing evidence for a link between inhibition and mathematics before discussing different types of inhibition task and different components of arithmetic. Finally, we introduce the present study.

\subsection{Inhibition and mathematics performance}

In the past 5 years there has been a steady increase in the number of studies that have explored the role of inhibition skills in mathematics performance. The majority of studies have employed correlational methods to explore the relationship between performance on tests of inhibition and concurrent mathematics achievement. For example, children's performance on experimental inhibition tasks are related to their school mathematics grades (Brock et al. 2009; Visu-Petra et al. 2011) as well as performance on standardized mathematics tests (Nayfield et al. 2013; St Clair-Thompson and Gathercole 2006). A smaller number of studies have found that inhibition predicts future success in mathematics (Blair and Razza 2007; Clark et al. 2010; Swanson 2011). Converging evidence for a link between inhibition and mathematics comes from studies that have compared the inhibition skills of different groups of children. Studies by Szucs et al. (2013), Wang et al. (2012) and Winegar (2013) found that children with identified mathematical learning difficulties performed more poorly on inhibition tasks than children with average performance in mathematics.

In contrast to these findings, a number of studies have failed to find evidence for a link between inhibition skills and mathematics: Waber et al. (2006) found weak relationships between experimental measures of inhibition and curriculum measures of mathematics performance; Miller et al. (2013) found that inhibition skills were not a unique predictor of mathematics performance and Monette et al. (2011) found that inhibition skills predicted future reading/ writing achievement but not future mathematics achievement. There is some evidence that inhibition skills may only be related to mathematics outcomes if shifting skills are not taken into account. Both Bull and Scerif (2001) and Van der Ven et al. (2012) found that measures of inhibition were no longer related to mathematics once shifting skills were included in the model. However, Espy et al. (2004) found that inhibition did predict mathematics even after controlling for both working memory and shifting skills.

To summarize, there is mixed evidence concerning the relationship between inhibition and mathematics performance. Although a number of studies have found a positive relationship, this appears to be more nuanced than originally proposed. Previous studies have been conducted with participants across a wide range of ages. This is an important consideration as inhibition skills mature and the nature of mathematics content changes with age, thus some of the inconsistencies outlined above may reflect changes in the role of inhibition skills across age. Two further factors may be important in explaining these inconsistencies: the type of inhibition task and the nature of the mathematics test. These will be considered in further detail below.

\subsection{Types of inhibition task}

Previous studies have employed a wide range of tasks to assess inhibition. These different tasks tap into varying aspects of inhibition skill. A distinction is commonly made between response inhibition and interference control (e.g. Nigg 2000). Interference control concerns the suppression of distracting information, either internal or external, which leads to an alternative non-desired response. The Stroop task is the best-known measure of interference control. In this task participants are required to focus on and respond to one aspect of a given stimulus (e.g. the colour of ink a word is written in) whilst ignoring other features of the stimulus (e.g. the word itself). Stroop tasks have frequently been employed in studies exploring the relationship of inhibition with mathematics performance (e.g. Bull and Scerif 2001; Lemaire and Lecacheur 2011; Monette et al. 2011; Navarro et al. 2011; St Clair-Thompson and Gathercole 2006; Szucs et al. 2013; Van der Ven et al. 2012; Visu-Petra et al. 2011).

A second form of inhibition is response inhibition. This concerns the suppression of a prepotent motor response and is often measured using Go/No-Go or Stop-signal tasks. In these tasks participants are required to frequently make one type of response unless they receive a signal to withhold the response. Inhibitory control performance is indexed by a failure to withhold the prepotent response. A smaller number of studies have used these types of tasks when exploring the relationship with mathematics achievement (De Weerdt et al. 2013; Monette et al. 2011; St Clair-Thompson and Gathercole 2006). At present there is evidence from different studies to suggest that both interference control and response inhibition are related to mathematics achievement (St Clair-Thompson and Gathercole 2006; Szucs et al. 2013); however, the relative importance of each type of inhibition remains unclear. Moreover, the mechanisms by which they support mathematics performance are likely to differ.

A further distinction that applies to both interference control and response inhibition tasks is between tasks that involve the inhibition of domain-relevant and 
domain-irrelevant information. It has been proposed that, rather than a single inhibitory system that is applied across all domains, there are multiple, domain-specific, inhibitory control systems (Egner 2008). Consequently, participants may show differing levels of inhibitory control according to the nature of the information they are being required to inhibit. More importantly here, mathematics achievement may be more strongly related to the inhibition of numerical information, rather than applying to inhibition skills more generally (Bull and Scerif 2001). To explore this question, multiple versions of inhibition tasks have been employed which involve the inhibition of either numerical or nonnumerical information. For example, alongside the standard colour-word stroop, which involves non-numerical information, studies have employed stroop tasks that involve the inhibition of numerically relevant information. For example Szucs et al. (2013), Wang et al. (2012), Zhang and Wu (2011), and Navarro et al. (2011) used a number-size stroop task in which participants are required to select the numerically highest digit whilst ignoring the size of the digits on the screen (e.g. 3 vs. 5). Bull and Scerif (2001) and Wang et al. (2012) have also made use of a number-quantity stroop task in which participants are required to name how many items are in a set while ignoring the digit itself (i.e. to respond "three" to the stimulus 555). Similarly, numerical and non-numerical versions of Go/No-Go tasks (De Weerdt et al. 2013) and random generation tasks (Winegar 2013) have also been developed. These alternative task versions have been used to explore the hypothesis that the relationship between inhibition and mathematics achievement is specific to the inhibition of numerically relevant information, with mixed results.

In favour of the domain-specific inhibition hypothesis, both Bull and Scerif (2001) and Navarro et al. (2011) found that only performance on the number-quantity version of the stroop task and not performance on the colour-word version correlated with mathematics achievement. Similarly, when comparing the performance of children with and without mathematics learning difficulties, both Szucs et al. (2013) and Wang et al. (2012) found that group differences were only significant for numerical stroop tasks rather than non-numerical versions. However, other studies have failed to find domain-specific effects with stroop tasks (Zhang and Wu 2011) or Go/No-Go tasks (De Weerdt et al. 2013).

In summary, there is some evidence to suggest that the content of inhibition tasks has an impact on the relationship with mathematics achievement. It is unclear, however, if this effect only arises in tasks involving the processing of Arabic digits or, alternatively, whether this holds for numerically relevant information more generally. Both the number-size and number-quantity versions of the stroop task, for which domain-specific effects have been observed, involve Arabic digits. Bull and Scerif (2001) suggest that, before conclusions about domain-specific numerical inhibition effects can be justified, versions of the stroop task with different types of numerical stimuli should be explored. An alternative task, which involves numerically relevant information but does not include Arabic digits, is the dot comparison task. In this task, which was originally developed to measure numerical magnitude processing, participants are shown pairs of dot arrays and are asked to select the more numerous array while ignoring the visual characteristics (e.g. dot size, density, area) of the arrays. Typically two types of trials are included: congruent trials, in which the more numerous array also has the larger visual characteristics, and incongruent trials, in which the less numerous array has larger visual characteristics. It has been proposed that solving incongruent trials of this task therefore has significant inhibitory control demands (Fuhs and McNeil 2013; Gilmore et al. 2013; Nys and Content 2012) and that the difference in performance on congruent and incongruent trials therefore provides a measure of inhibition skills. In line with this, Szucs and colleagues (2013) found that children with mathematics learning difficulties had larger congruency effects on a dot comparison task than controls.

Congruency effects on a dot comparison task therefore provide a measure of inhibition in a numerical context without the use of Arabic digits themselves. Performance on this task can be contrasted with an equivalent task involving non-numerical information. A suitable task here is the animal-size stroop task that has been used by Szucs et al. (2013). In this task participants are shown two animal images and are asked to select the animal that is larger in real life, while ignoring the size of the images on the screen. Again, participants see both congruent trials, in which the animal that is larger in real life is also larger on screen, and incongruent trials, in which the animal that is smaller in real life is larger on screen. Performance on incongruent trials of both the dot comparison and animal stroop tasks involve ignoring the superficial task-irrelevant visual characteristics of the stimuli, but the tasks differ in whether the relevant processing is numerical or not. These tasks therefore allow us to test whether both domain-specific (i.e. numerical) and domain-general (i.e. non-numerical) measures of interference control are related to mathematics achievement.

\subsection{Components of arithmetic}

A second factor that may help to explain the conflicting results surrounding the relationship between inhibition skills and mathematics performance concerns the nature of mathematics involved. Rather than being a unitary skill, mathematics is a multi-componential construct. Not only can different domains be identified, for example arithmetic, 
algebra, or geometry, but researchers have also identified specific components that cut across these domains (see review by Rittle-Johnson and Schneider, 2014). Researchers typically discriminate factual knowledge, procedural skill and conceptual understanding. Factual knowledge comprises memorized number facts, for example the addition and multiplication tables. Procedural skill concerns the accurate and efficient execution of operations (e.g. "carrying' when adding above 10) and can be thought of as 'knowing how'. Conceptual understanding, on the other hand, is knowledge of the principles and relationships that underlie mathematics (e.g. knowing that addition is the inverse of subtraction) or 'knowing why'. It is well established that there are complex relationships among these components (Baroody and Dowker, 2003), which are not hierarchically ordered. Furthermore, individuals differ in their profile of performance across these components, and may have strengths in one component but not others (Dowker 2005) suggesting that these components rely on differential sets of skills.

The vast majority of previous research exploring the role of inhibition has involved general standardized or curriculum tests of mathematics, which do not capture these individual elements. However, we need to move beyond these general tests of mathematics to allow the processes by which inhibition supports mathematics performance to be understood. It is likely that the precise mechanisms by which good inhibition skills support mathematics performance differ for factual, procedural and conceptual knowledge (Cragg and Gilmore, 2014).

Considering procedural skills first, there is evidence to suggest that inhibition is important in suppressing inefficient, but well rehearsed, strategies in favour of more efficient or new strategies. Lemaire and Lecacheur (2011) found that children with better inhibitory control made more use of the most efficient strategy to solve arithmetic problems compared to children with lower levels of inhibitory control. Thus, children with lower levels of inhibitory control may be able to generate alternative strategies without difficulty, but are less able to switch between strategies flexibly in response to context (c.f. Bull and Scerif 2001 performance on Wisconsin Card Sorting Task). The ability to make adaptive strategy choices is a characteristic of children who are proficient with mathematics (Torbeyns et al. 2006) and thus this mechanism might explain the general advantage in mathematics shown by children with good inhibition skills.

Turning next to conceptual understanding, Robinson and Dubé (2013) found that children with lower levels of inhibitory control made more use of mixed procedural and conceptual approaches than children with higher levels of inhibitory control, and suggested that poor inhibition was associated with an inability to suppress procedural in favour of conceptual approaches. Consistently favouring procedural over conceptual strategies could interfere with the developmental of rich conceptual understanding. Similarly, computational models of conceptual development have proposed that inhibition is required to shift attention away from procedural solutions to allow underlying numerical relationships to be identified (Siegler and Araya, 2005).

Finally, it has also been suggested that inhibition is important for correctly retrieving known number facts from memory due to the way in which number facts are stored. It has been proposed that addition and multiplication number facts are stored in an associative network (Campbell et al. 2011). As a result, the solutions to alternative problems can interfere with the retrieval of a desired solution and inhibition is required to suppress these alternatives. This can either occur from interference of "neighbouring" solutions (e.g. " 42 " might interfere with retrieving the answer to " $6 \times 8$ ") or alternative operations (e.g. " 15 " might interfere with retrieving the answer to " $5+3$ ").

There is some evidence to support the hypothesis that inhibition skills are differentially related to multiple components of mathematics. Winegar (2013) found that, for children in Grades 3 and 4, inhibition skills were related to arithmetic word problem solving but not to calculation skills. Similarly, in a study of preschool children Lan et al. (2011) found that inhibition was an independent predictor of counting but not calculation skills. These differences may reflect a stronger role of inhibition in executing sequential procedures rather than recalling number facts.

These studies provide some evidence to suggest that inhibition skills play a varying role in different components of mathematics; however, these relationships have yet to be systematically explored in a single study. Furthermore, the nature of these relationships may change as children mature and develop more advanced knowledge of mathematics. The domain-general processing demands of, for example number fact knowledge, will change as children move from fragile memory of a small set of number facts through to having secure memory of a complete number fact table. It is important, therefore, to consider how these relationships change over development.

\subsection{The present study}

Here, we present a study that explores the relationship between inhibition skills and mathematics performance in detail by addressing three key research questions. First we explore whether inhibition skill measured in both numerical and non-numerical contexts is related to overall mathematics performance. Secondly, we consider whether inhibition skill is related to the individual components of factual, procedural and conceptual knowledge of mathematics as well as overall achievement. Finally, we test whether the 
relationship between inhibition skill and different components of mathematics is consistent across ages. To answer these questions, we administered numerical (dot comparison) and non-numerical (animal stroop) versions of inhibition tasks as well as tests of individual components of arithmetic knowledge and overall mathematics achievement to adult and child participants.

\section{Method}

\subsection{Participants}

A total of 67 11- to 12-year-olds in school Year 7 ( $M=12.25$ years, SD $=0.35 ; 32$ female $), 6713$ - to 14 -year-olds in school Year $9(M=14.23$ years, $\mathrm{SD}=0.3$; 37 female) and 75 young adults $(M=21.28$ years, $\mathrm{SD}=1.69 ; 48$ female) took part in the study. The children attended secondary schools in predominantly Caucasian, average socio-economic status neighbourhoods of Nottingham, UK. Informed consent was received for all children, who were given a certificate for taking part. The adults were students from Loughborough University and the University of Nottingham. They gave informed consent and received a small inconvenience allowance. All participants were part of a larger study examining the general thinking skills involved in learning and doing mathematics. Ethical approval was obtained from the Loughborough University Ethics Committee.

\subsection{Equipment and materials}

The arithmetic and inhibition tasks were created using PsychoPy software (Peirce 2007) and run on an HP laptop computer. Responses were made using an external numeric keypad. For the mathematics tasks, the experimenters recorded response times for child participants by pressing a key immediately as children began to give their answer.

\subsection{Tasks}

\subsubsection{Mathematics achievement test}

The Mathematics Reasoning and Numerical Operations subtests of the Wechsler Individual Achievement Test (Wechsler 2005) were administered following the standard procedure. These tests provide a broad assessment of curriculum-relevant mathematics achievement. The Mathematics Reasoning subtest assesses performance on a series of verbally and visually presented word problems covering problem solving, geometry, measurement, reasoning, graphs and statistics. The Numerical Operations subtest is a pencil and paper test of arithmetic and algebra. We used
Mathematics Composite standard scores, which combine scores across the two subtests.

\subsubsection{Factual knowledge task}

This task assessed participants' knowledge of number facts. On each trial an arithmetic problem was presented on screen for $3 \mathrm{~s}$ and participants were asked to retrieve the result without mental calculation. The participants were instructed to give their answer verbally, at which point the experimenter pressed a key and inputted by the answer. Participants were instructed to say "I don't know" if they could not retrieve the answer.

Participants completed four practice trials and then 12 experimental trials in random order. An additional four easy 'motivational trials' were intermixed with the experimental trials but were not included in the analysis. Following pilot testing, we selected a different set of items for each age group (11-12, 13-14 years and adults) to ensure that performance was not at floor or ceiling level in any group. The problems given to the secondary school students were composed of addition and subtraction operations only. The problems for the 11- to 12-year-olds involved single-digit numbers, and the problems for the 13- to 14-year-olds were composed of one single-digit number and one double-digit number. The trials given to adult participants were composed of addition, subtraction, multiplication and division operations involving one single-digit number and one double-digit number. The measure of performance was the proportion of items answered correctly within the 3-s presentation time. Higher scores indicated better performance.

\subsubsection{Procedural skills task}

This task assessed the efficiency with which participants could accurately perform arithmetic procedures. On each trial an arithmetic problem was presented on screen and participants were instructed to solve it using any mental method they preferred. To ensure that younger participants understood that any strategy was acceptable in this task, prior to starting the task participants were shown pictures representing different strategies (i.e. counting in your head, counting on fingers, decomposition, and retrieval). The experimenter described the strategies and told participants that any of these strategies, or others, could be used to solve the task.

Participants were given four practice trials and then 10 (11-12 years age group) or 12 (13-14 years age group and adults) experimental trials. Following pilot testing, the operations were designed to be age appropriate, and of a difficulty level where retrieval would be unlikely. The trials given to 11- to 12-year-olds were composed of addition and subtraction operations and the trials given to 13- to 
Fig. 1 Example stimuli for a the non-numerical inhibition task and $\mathbf{b}$ the numerical inhibition task. Panels on the left depict a congruent trial and panels on the right depict an incongruent trial
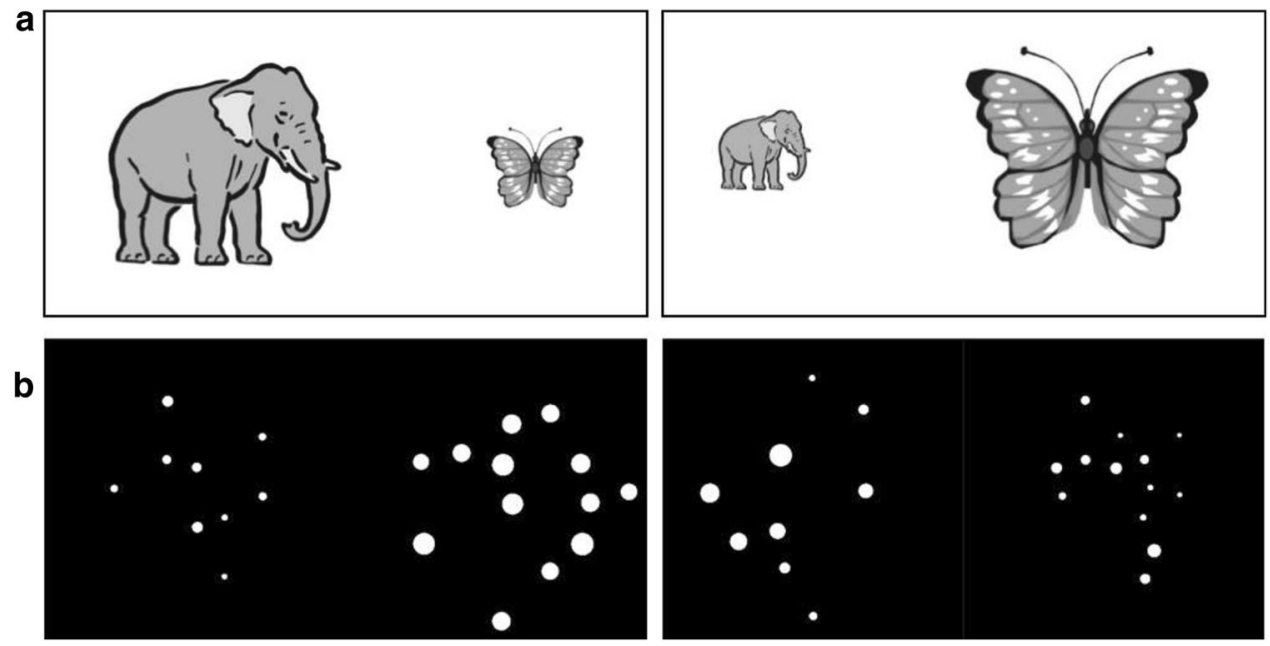

14-year-olds and adult were composed of addition, subtraction, multiplication and division operations. The problems for each age group involved a mix of single and doubledigit numbers. The items in each version were presented in one of two orders counterbalanced across participants.

The participants were instructed to give their answer verbally, at which point the experimenter pressed a key and inputted the answer. After each answer, the participants were questioned on their strategy use. The measure of performance on this task was median response time (RT) for correctly answered trials. For ease of analysis, scores were multiplied by -1 so that higher scores indicated better performance.

\subsubsection{Conceptual knowledge task}

This task assessed participants' understanding of conceptual principles underlying arithmetic. On each trial an arithmetic problem with the correct answer was presented on the screen. Once this was read, the experimenter pressed 'return' and a second, unsolved operation appeared below the first problem. The participants were asked to state whether or not the first problem could help solve the second problem, and then were asked to explain how. They were discouraged from actually solving the second problem. Eighteen of the 30 problem pairs were related. The pairs of problems were related by the subtraction-complement principle (e.g. $113-59=54$ and $113-54=$ ), inverse operations (e.g. $74+57=131$ and $131-74=$ ), and associative operations (e.g. $87-54=33$ and $87-34-20=$ ). Twelve of the problem pairs were unrelated but were designed to look superficially similar to the related problems (e.g. $69+54=123$ and $69+45=$ ). The operations were designed to be difficult to solve mentally, to discourage the participants from attempting to do so.
Participants were given four practice trials and 30 experimental trials. As with the other arithmetic tasks, a different set of problems were used for each age group. The trials given to the 11- to 12-year-olds were composed of addition and subtraction problems involving two operands of two and three digit numbers. The trials given to the 13- to 14-year-olds and adults were composed of addition and subtraction problems involving two or three operands of double-digit numbers, as well as some multiplication and division problems involving single and double-digit numbers. The items in each task version were presented in one of two orders counterbalanced across participants.

Participants gave their response verbally and the experimenter recorded this. The measure of performance was the proportion of trials for which the presence or absence of a relationship was correctly identified. Higher scores indicated better performance.

\subsubsection{Non-numerical inhibition task}

To assess participants' ability to inhibit irrelevant information in a non-numerical context, we used an animal-size stroop task (based on Szucs et al. 2013). On each trial two animal pictures were presented on the screen. One animal was selected from a set of large animals (e.g. a bear, gorilla, and giraffe) and the other animal was selected from a set of small animals (e.g. an ant, rabbit, and mouse). The participants' task was to identify which animal was the larger in real life. On each trial, one animal image was presented with an area on screen four times larger than the other image. On congruent trials the animal that was larger in real life was also the larger image on the screen, and on incongruent trials the animal that was smaller in real life was the larger image on the screen (see Fig. 1a). Participants were required to ignore the size of the images on the screen and to respond based on the size in real life only. On 
Table 1 Descriptive statistics for performance on the experimental mathematics tasks

\begin{tabular}{|c|c|c|c|c|c|c|}
\hline \multirow[t]{2}{*}{ Task } & \multicolumn{2}{|c|}{ Age $11-12$ years } & \multicolumn{2}{|c|}{ Age $13-14$ years } & \multicolumn{2}{|l|}{ Adults } \\
\hline & Mean (SD) & Range & Mean (SD) & Range & Mean (SD) & Range \\
\hline Factual & $0.65(0.27)$ & $0.1-1.0$ & $0.67(0.26)$ & $0.2-1.0$ & $0.83(0.16)$ & $0.3-1.0$ \\
\hline Procedural & $9.4(3.6)$ & $3.5-18.3$ & $8.8(3.4)$ & $3.6-17.9$ & $6.8(2.5)$ & $2.5-14.8$ \\
\hline Conceptual & $0.76(0.17)$ & $0.4-1.0$ & $0.81(0.15)$ & $0.4-1.0$ & $0.92(0.07)$ & $0.73-1.0$ \\
\hline
\end{tabular}

Factual score is the proportion of number facts correctly produced within $3 \mathrm{~s}$. Procedural score is the median RT in seconds for solving arithmetic problems. Conceptual understanding is the proportion of correct responses to problem pairs

each trial, the images were presented on screen and participants responded as quickly as possible by pressing one of two buttons on the keyboard that corresponded to the side of the screen with the larger animal.

Participants completed four experimental blocks each containing 48 trials in random order. In two of the experimental blocks $75 \%$ of the trials were congruent and $25 \%$ were incongruent and in two of the experimental blocks $75 \%$ of the trials were incongruent and $25 \%$ were congruent. The blocks were presented in counterbalanced order and participants had the opportunity to take breaks during the task as needed.

Prior to commencing the task participants were shown each of the animal images in one size and asked whether the animal was large or small in real life to ensure they had the necessary real-world knowledge to perform the task. All participants completed this without problem.

Median RTs for correctly solved trials were calculated for the congruent and incongruent trials (collapsing across blocks). Inhibition score was the difference in RT for congruent and incongruent trials. Larger differences indicate lower levels of inhibitory control.

\subsubsection{Numerical inhibition task}

Participants completed 6 practice trials and 80 experimental trials in random order. They were given breaks during the task as needed. Mean accuracy was calculated for the congruent and incongruent trials. Inhibition score was the difference in accuracy for congruent and incongruent trials. Larger differences indicate lower levels of inhibitory control.

To assess participants' ability to inhibit irrelevant information in a numerical context, we used a dot comparison task. On each trial the participants were shown two sets of white dots on a black screen and were instructed to identify which set had the highest number of dots. The dots were created using an adapted version of the matlab script provided by Gebuis and Reynvoet (2011). This method produced two types of trials. On congruent trials the more numerous array has larger dots and the array encompasses a larger area. On incongruent trials the more numerous array has smaller dots and the array encompasses a smaller area (see Fig. 1b). Participants were required to ignore the size of the dots and the array on the screen and to respond based on the number of dots only. The number of dots in each array ranged from 5 to 28 and the ratio between the number of dots ranged from 0.5 to 0.8

\subsection{Procedure}

Each participant was tested individually in a 2-h session. In addition to the tasks described above they completed further tasks to assess their mathematical and executive function skills. The tasks were presented in one of two orders counterbalanced across participants. The children were all tested in quiet rooms away from the classroom and the adults were tested in a quiet laboratory.

\subsection{Data preparation}

The content of the arithmetic tasks varied for each age group to prevent floor or ceiling effects on any tasks. As a result it was not appropriate to use raw scores in analyses involving multiple age groups. We therefore transformed raw scores on the arithmetic and inhibition tasks to $z$-scores within each age group and used these in the subsequent analyses. Similarly, we used age-standardized scores on the mathematics achievement test. Outliers that were more than three times the standard deviation from the mean were excluded from individual tasks (one participant from the non-numeric inhibition task and one participant from the mathematics achievement task).

\section{Results}

\subsection{Task performance}

Descriptive statistics for performance on the mathematics tasks is provided in Table 1 . There was a range of performance on each task and thus the items selected for each age group were appropriate.

Mean performance on the congruent and incongruent trials of the two inhibition tasks is provided in Fig. 2. 
Fig. 2 Congruency effects on the non-numerical and numerical inhibition tasks



Table 2 Hierarchical regression models exploring the relationship between mathematics achievement and numerical vs. non-numerical inhibition scores

\begin{tabular}{llllll}
\hline Model & Step & Variable & $\beta$ & $\Delta R^{2}$ & Sig. $\Delta R^{2}$ \\
\hline 1 & 1 & Non-numerical inhibition & $-0.177^{*}$ & 0.0310 .016 \\
& 2 & Non-numerical inhibition & -0.097 & 0.049 & 0.002 \\
& & Numerical inhibition & $-0.234^{* *}$ & & \\
2 & 1 & Numerical inhibition & $-0.268^{* * *}$ & $0.072<.001$ \\
& 2 & Numerical inhibition & $-0.234^{* *}$ & 0.008 & 0.204 \\
& & Non-numerical inhibition & -0.097 & & \\
\hline
\end{tabular}

Dependent variable $=$ WIAT mathematics composite standard score; significance of $\beta$ weights: $* p<.05, * * p<.01$, *** $p<.001$. Model $R^{2}=0.08$

These data were explored via two mixed-design ANOVAs with congruency (congruent, incongruent) as repeatedmeasures factor and age group (11-12, 13-14 years, adult) as between-groups factor. Dependent variables were RTs for the non-numerical inhibition task and accuracy for the numerical inhibition task.

For the non-numerical inhibition task there was a significant main effect of age group $(F(2,205)=35.1, p<.001$, $\left.\eta_{\mathrm{p}}^{2}=0.26\right)$ with RTs faster for adults than for children aged 13-14 years $(p<.001)$ and faster for children aged 13-14 years than $11-12$ years $(p=.001)$. There was also a significant main effect of congruency $(F(1,205)=805.9$, $\left.p<.001, \eta_{\mathrm{p}}^{2}=.80\right)$ with RTs faster for congruent than for incongruent trials. There was no interaction between age group and congruency $(F(2,205)=1.2, p=.302$; see Fig. 2).

For the numerical inhibition task there was a significant main effect of age group $(F(2,206)=21.7, p<.001$, $\left.\eta_{\mathrm{p}}^{2}=.17\right)$ with accuracy higher for adults than children aged 13-14 years $(p=.003)$ and higher for children aged $13-14$ years than $11-12$ years $(p=.006)$. There was also a significant main effect of congruency $(F(1,206)=204.4$, $p<.001, \eta_{\mathrm{p}}^{2}=.50$ ) with accuracy higher for congruent than for incongruent trials. There was also a significant interaction between age group and congruency $(F(2,206)=4.8$, $p=.009, \eta_{\mathrm{p}}^{2}=.05$; see Fig. 2). Bonferroni-corrected post hoc tests revealed significant congruency effects for all three age groups (all $p \mathrm{~s}<.001$ ). Thus, the task was appropriate to assess inhibitory control in each age group.

\subsection{The relationship between mathematics achievement and numerical vs. non-numerical inhibition}

The first step in the analysis was to explore the relationship between performance on the two inhibition tasks with overall mathematics achievement. Numerical inhibition score was moderately correlated with mathematics achievement $(r=-.271, p<.001)$. Non-numerical inhibition score showed a small significant correlation with mathematics achievement $(r=-.177, p=.016)$. Thus, participants with a smaller difference in accuracy on congruent and incongruent dot comparison trials, or who had a smaller difference in RT on congruent and incongruent animal stroop trials tended to have higher levels of mathematics achievement. Inhibition scores from the two tasks were also significantly correlated $(r=.345, p<.001)$.

We then explored whether numerical and non-numerical inhibition had independent relationships with mathematics achievement. We conducted two linear regression models with mathematics achievement as dependent variable and each type of inhibition score as independent variables. In the first model, non-numerical inhibition score was added in the first step, and numerical inhibition score was added in the second step. In the second model these steps were reversed. As shown in Table 2, these models produced different patterns of results. Numerical inhibition score was a significant predictor, over and above non-numerical inhibition score, whereas non-numerical inhibition score added nothing to the model after numerical inhibition score. Thus, 
these inhibition tasks are not equivalent and numerical inhibition has an independent relationship with mathematics over and above general inhibition. In the remainder of the analyses, therefore, numerical inhibition scores alone will be used to explore the relationship between inhibition and multiple components of arithmetic.

\subsection{The relationship between inhibition and multiple components of arithmetic}

We next explored whether inhibition skill had a differing relationship with measures of factual, procedural and conceptual knowledge of arithmetic. Zero-order correlations revealed small, but significant, correlations between numerical inhibition skill and each measure of arithmetic (factual knowledge, $r=-.193, p=.005$; procedural skill, $r=-.267, p<.001$; conceptual understanding, $r=-.188$, $p=.006$ ).

To discover whether these correlations represented independent relationships, we conducted a linear regression with inhibition score as the dependent variable and factual, procedural and conceptual arithmetic scores as the independent variables. This allowed us to test the relationship between inhibition and each component of mathematics simultaneously. This revealed that factual knowledge was not a significant predictor $(0=0.045, p=.654)$, procedural skill was a significant predictor $(\beta=-0.260, p=.007)$ and conceptual understanding was a marginally significant predictor $(\beta=-0.134, p=.066)\left(\operatorname{model} R^{2}=0.085\right)$. Therefore, inhibition appears to be most strongly related to procedural skills and, to a lesser extent, related to conceptual understanding.

Finally, we explored whether the relationship between inhibition skill and each component of arithmetic was similar in participants who were in secondary school, and therefore still learning mathematics, compared with adults. We repeated the above linear regression separately for the adolescents and adults and examined the $\beta$ weights for the different components of arithmetic. As shown in Table 3, there appears to be transition over development. At age 11-14 years, only procedural skill was significantly related to inhibition skill, whereas for the group of young adults only conceptual understanding was significantly related to inhibition skill.

\section{Discussion}

In this study, we investigated for the first time the relationship between inhibition and multiple components of mathematics. In line with an increasing body of previous evidence (e.g. Brock et al. 2009; Bull and Scerif 2001; Kroesbergen et al. 2009) we found that there was a
Table 3 The relationship between inhibition skill and multiple components of arithmetic in children and adults

\begin{tabular}{lll}
\hline Independent variable & Age 11-14 & Young adults \\
\hline Factual knowledge & $\beta=0.043, p=.740$ & $\beta=0.069, p=.665$ \\
Procedural skill & $\beta=-0.346$, & $\beta=-0.117, p=.442$ \\
& $p=.006^{* *}$ & \\
Conceptual under- & $\beta=-0.049, p=.588$ & $\beta=-0.293, p=.019^{*}$ \\
$\quad$ standing & & \\
\hline
\end{tabular}

The table reports beta weights (and significance levels) for each independent variable in linear regressions with inhibition skill as dependent variable conducted separately for each group

$R^{2}=0.11$ (11- to 14-year-olds) $R^{2}=0.10$ (adults); $* p<.05$, $* * p<.01$

significant relationship between inhibition skill and mathematics performance. However, our use of both numerical and non-numerical inhibition tasks as well as tests of multiple components of arithmetic allowed a more nuanced picture to emerge. First, we found evidence for a stronger relationship between mathematics and performance on a numerical, compared to a non-numerical, inhibition task. Secondly, we found that inhibition skills were an independent predictor of procedural skills in the group of secondary school children and conceptual understanding in the adult group. There was no significant independent relationship with factual knowledge in either group. Below we consider the novel contributions of this study and their theoretical implications.

We introduced a novel technique to assess inhibition skills in a numerical context-the dot comparison task. Previously, overall performance on this task has been used to explore the nature of numerical magnitude representations. However, recent findings have demonstrated that incongruent trials, but not congruent trials carry a heavy inhibition load (Fuhs and McNeil 2013; Gilmore et al. 2013; Nys and Content 2012; Szucs et al. 2013). As a result, difference in performance on congruent and incongruent trials provides a measure of inhibition skill. We have shown here that this measure of inhibition is related to both performance on a standard inhibition task, and to overall mathematics achievement. Importantly, this measure of inhibition predicted mathematics performance even after accounting for performance on a non-numerical measure of inhibition. This suggests that there is something specific about the ability to ignore irrelevant numerical information that is particularly important for mathematics performance. Our findings go beyond previous evidence for a domainspecific effect (Bull and Scerif 2001; Navarro et al. 2011; Szucs et al. 2013; Wang et al. 2012) and show that this is not unique to tasks involving Arabic digit stimuli.

Why might there be a distinction between numerical and non-numerical inhibition skills? There is some evidence that 
domain-specific effects are similarly found for the relationship between working memory and mathematics (DeStefano and LeFevre 2004; David 2012). Bull and Scerif (2001) suggest that evidence of domain-specific inhibition effects could be related to differences in storage capacity across varying types of information (see also Szucs et al. 2013). Further research is needed that incorporates both measures of inhibition skill and working memory to better understand the nature of these domain-specific effects.

To our knowledge, this study was the first to systematically investigate the relationship between inhibition and separate measures of factual, procedural and conceptual knowledge of mathematics. Evidence for both a differential relationship between inhibition and various components, and the changing nature of these relationships over development helps to pinpoint the types of mechanisms by which inhibition may act. In line with previous research (e.g. Lemaire and Lecacheur, 2011), we found a relationship between inhibition skill and procedural efficiency in school-aged children. Children who had better inhibition performance were able to execute accurate solutions more quickly. This may have reflected a role for inhibition either via more efficient execution of strategies or via the selection of more advanced strategies (Lemaire and Lecacheur, 2011). We did not find a relationship between procedural efficiency and inhibition in adults, which might suggest that the more likely explanation is that inhibition supports the selection of more efficient strategies, given that adults show less variation in strategy selection compared with children. In keeping with this, Khng and Lee (2009) found that inhibition skill, measured with a battery of tasks, was related to the selection of sophisticated solution strategies, in preference to well-learned prepotent arithmetic strategies when solving algebraic word problems.

In contrast to previous research (Robinson and Dubé 2013), we found no relationship between inhibition skills and conceptual understanding in children, but did find a relationship for adult participants. Robinson and Dubé's study explored the application of conceptually based strategies when actively solving problems, whereas our conceptual task measured the ability to identify conceptual relationships themselves. The problems included in our conceptual task were designed to be at the upper limit of what participants could solve procedurally and therefore provide a purer measure of conceptual understanding. The mean scores for each group of participants on this task (Table 1) suggest that we were less successful in making the problems difficult to solve procedurally for the adults compared to the younger participants. Thus, it is possible that inhibition may be important in cases where a procedural alternative is possible (e.g. Robinson and Dubé's study or the adults in our study) and less important when drawing on conceptual knowledge per se (e.g. the children in our study). Further research with a range of conceptual measures is needed to further explore this pattern. In particular, it would be valuable to explore these relationships in a variety of mathematical domains, to establish the stability of the relationships identified here. If confirmed, our findings imply that it may be beneficial for children to be given the opportunity to develop conceptual knowledge in tasks where alternative procedural approaches are not possible as this lessens the inhibitory control load.

It is somewhat surprising that no evidence was found for a relationship between inhibition and factual knowledge. Previous research has suggested that inhibition is important for suppressing related but incorrect solutions when retrieving number facts (Campbell et al. 2011). There are several possible explanations of why we failed to find a relationship between inhibition and factual knowledge. First, previous explorations of interference effects in fact retrieval have employed experimental, rather than correlational, designs. It is possible that correlational approaches are not sensitive enough to detect this effect. Alternatively, this might be related to the domain-specificity effect discussed earlier and fact retrieval may only be related to inhibition involving digits. Inhibition measured on number-size (e.g. Szucs et al. 2013) or number-quantity (e.g. Bull and Scerif 2001) stroop tasks, which involve Arabic digits, may therefore show a different pattern. Finally, this may reflect different types of inhibition. Our inhibition tasks were measures of interference control, however, fact retrieval is a rapid process and it is plausible that links between factual knowledge and inhibition are only revealed when response inhibition tasks (e.g. Stop-signal tasks) are employed. In this study, we have begun to unpick the nuances of the relationship between inhibition and different components of mathematics, but further research involving a variety of inhibition tasks is required to explore these potential explanations.

We have shown here that performance on an inhibition task is related to performance on concurrent measures of mathematics performance. The correlational design used in this study cannot identify whether the mechanisms by which inhibition relates to mathematics operate over a short or long timeframe. Inhibition skills may be related to mathematics performance because inhibition is involved in the online performance of mathematics procedures or alternatively inhibition may be involved in the learning of new mathematics material. In fact as the examples above suggest, we believe that inhibition is likely to be involved in both the learning of new material (e.g. identifying conceptual regularities) and the online performance of mathematics procedures (e.g. inhibiting a left-to-right solution strategy in favour of a more efficient approach when solving arithmetic problems). In order to tease apart these differences, research needs to move beyond the correlational designs that predominate in this literature and employ experimental and longitudinal studies. 
To conclude, we found that inhibition skills are related to mathematics performance but the pattern of relationships changes across forms of inhibition, components of mathematics and age. This demonstrates that, rather than representing a global effect, the role of inhibition in mathematics results from a range of specific mechanisms. Future studies should employ specific, as opposed to general, measures of both inhibition and mathematics in order to advance our understanding of these complex relationships. Inhibition is one of many general cognitive skills that have an impact on children's mathematics achievement. Progress in understanding the way in which these cognitive skills support or interfere with children's learning will help to reveal why many children struggle with mathematics and provide avenues to explore future approaches to intervention.

Open Access This article is distributed under the terms of the Creative Commons Attribution License which permits any use, distribution, and reproduction in any medium, provided the original author(s) and the source are credited.

\section{References}

Baroody, A. J., \& Dowker, A. (2003). The development of arithmetic concepts and skills: Constructing adaptive expertise. Mahwah: Erlbaum.

Blair, C., \& Razza, R. P. (2007). Relating effortful control, executive function, and false belief understanding to emerging math and literacy ability in kindergarten. Child Development, 78, 647-663. doi:10.1111/j.1467-8624.2007.01019.x.

Brock, L. L., Rimm-Kaufman, S. E., Nathanson, L., \& Grimm, K. J. (2009). The contributions of 'hot' and 'cool' executive function to children's academic achievement, learning related behaviours, and engagement in kindergarten. Early Childhood Research Quarterly, 24, 713-721. doi:10.1016/j.ecresq.2009.06.001.

Bull, R., \& Lee, K. (2014). Executive functioning and mathematics achievement. Child Development Perspectives, 8, 36-41. doi:10.1111/cdep.12059.

Bull, R., \& Scerif, G. (2001). Executive functioning as a predictor of children's mathematics ability: Inhibition, switching, and working memory. Developmental Neuropsychology, 19, 273-293. doi:10.1207/S15326942DN1903 3.

Campbell, J. I. D., Dowd, R. R., Frick, J. M., McCallum, K. N., \& Metcalfe, A. W. S. (2011). Neighborhood consistency and memory for number facts. Memory \& Cognition, 39, 884-893.

Clark, C. A., Pritchard, V. E., \& Woodward, L. J. (2010). Preschool executive functioning abilities predict early mathematics achievement. Developmental Psychology, 46, 1176-1191. doi:10.1037/ a0019672.

Cragg, L., \& Gilmore, C. (2014). Skills underlying mathematics: The role of executive function skills in the development of mathematics proficiency. Trends in Neuroscience and Education, 3, 63-68. doi:10.1016/j.tine.2013.12.001.

David, C. V. (2012). Working memory deficits in Math learning difficulties: A meta-analysis. International Journal of Developmental Disabilities, 58, 67-84. doi:10.1179/2047387711Y.0000000007.

De Weerdt, F., Desoete, A., \& Roeyers, H. (2013). Behavioural inhibition in children with learning disabilities. Research in Developmental Disabilities, 34, 1998-2007. doi:10.1016/j. ridd.2013.02.020.
DeStefano, D., \& LeFevre, J. (2004). The role of working memory in mental arithmetic. European Journal of Cognitive Psychology, 16, 353-386. doi:10.1080/09541440244000328.

Dowker, A. (2005). Individual differences in arithmetic: Implications for psychology, neuroscience and education. London: Psychology Press.

Egner, T. (2008). Multiple conflict-driven control mechanisms in the human brain. Trends in Cognitive Sciences, 12, 374-380. doi:10.1016/j.tics.2008.07.001.

Espy, K. A., McDiarmid, M. M., Cwik, M. F., Stalets, M. M., Hamby, A., \& Senn, T. E. (2004). The contribution of executive functions to emergent mathematic skills in preschool children. Developmental Neuropsychology, 26, 465-486. doi:10.1207/ s15326942dn2601_6.

Fuhs, M. W., \& McNeil, N. M. (2013). ANS acuity and mathematics ability in preschoolers from low-income house: Contributions of inhibitory control. Developmental Science, 16, 136-148. doi:10.1111/desc.12013.

Gebuis, T., \& Reynvoet, B. (2011). Generating nonsymbolic number stimuli. Behavior Research Methods, 43, 981-986. doi:10.3758/ s13428-011-0097-5.

Gilmore, C., Attridge, N., Clayton, S., Cragg, L., Johnson, S., Marlow, N., et al. (2013). Individual differences in inhibitory control, not non-verbal number acuity, correlate with mathematics achievement. PLoS ONE, 8, e67374. doi:10.1371/journal.pone.0067374.

Khng, K. H., \& Lee, K. (2009). Inhibiting interference from prior knowledge: Arithmetic intrusions in algebra word problem solving. Learning and Individual Differences, 19, 262-268. doi:10.1016/j.lindif.2009.01.004.

Lan, X., Legare, C. H., Ponitz, C. C., Li, S., \& Morrison, F. J. (2011). Investigating the links between the subcomponents of executive function and academic achievement: A cross-cultural analysis of Chinese and American Preschoolers. Journal of Experimental Child Psychology, 108, 677-692. doi:10.1016/j.jecp.2010.11.001.

LeFevre, J.-A., Fast, L., Skwarchuk, S.-L., Smith-Chant, B. L., Bisanz, J., Kamawar, D., \& Penner-Wilger, M. (2010). Pathways to mathematics: longitudinal predictors of performance: Pathways to mathematics. Child Development, 81(6), 1753-1767. doi:10.1111/j.1467-8624.2010.01508.x.

Lemaire, P., \& Lecacheur, M. (2011). Age-related changes in children's executive function and strategy selection: A study in computational estimation. Cognitive Development, 26, 282-294. doi:10.1016/j.cogdev.2011.01.002.

Miller, M. R., Müller, U., Giesbrecht, G. F., Carpendale, J. I., \& Kerns, K. A. (2013). The contribution of executive function and social understanding to preschoolers' letter and math skills. Cognitive Development, 28, 331-349. doi:10.1016/j. cogdev.2012.10.005.

Monette, S., Bigras, M., \& Guay, M. C. (2011). The role of executive functions in school achievement at the end of Grade 1. Journl of Experimental Child Psychology, 109, 158-173. doi:10.1016/j. jecp.2011.01.008.

Navarro, J. I., Aguilar, M., Alcade, C., Ruiz, G., Marchena, E., \& Menacho, I. (2011). Inhibitory processes, working memory, phonological awareness, naming speed, and early arithmetic achievement. The Spanish Journal of Psychology, 14, 580-588. doi:10.5209/rev_SJOP.2011.v14.n2.6.

Nayfield, I., Fuccillo, J., \& Greenfield, D. B. (2013). Executive functions in early learning: Extending the relationship between executive functions and school readiness to science. Learning and Individual Differences, 26, 81-88. doi:10.1016/j. lindif.2013.04.011.

Nigg, J. T. (2000). On inhibition/disinhibition in developmental psychopathology: Views from cognitive and personality psychology and a working inhibition taxonomy. Psychological Bulletin, 126, 220-246. doi:10.1037/0033-2909.126.2.220. 
Nys, J., \& Content, A. (2012). Judgments of discrete and continuous quantity in adults: Number counts! The Quarterly Journal of Experimental Psychology, 65, 675-690. doi:10.1080/17470218.2 011.619661.

Parsons, S., \& Bynner, J. (2005). Does numeracy matter more?. London: National Research and Development Centre for Adult Literacy and Numeracy.

Peirce, J. W. (2007). PsychoPy-Psychophysics software in Python. Journal of Neuroscience Methods, 162, 8-13. doi:10.1016/j. neumeth.2006.11.017.

Raghubar, K. P., Barnes, M. A., \& Hecht, S. A. (2010). Working memory and mathematics: A review of developmental, individual difference, and cognitive approaches. Learning and Individual Differences, 20, 110-122. doi:10.1016/j.lindif.2009.10.005.

Rittle-Johnson, B. \& Schneider, M. (2014). Developing conceptual and procedural knowledge of mathematics. In R. Kadosh \& A. Dowker (Eds.), Oxford Handbook of Numerical Cognition. Oxford: Oxford University Press.

Robinson, K. M., \& Dubé, A. K. (2013). Children's additive concepts: Promoting understanding and the role of inhibition. Learning and Individual Differences, 23, 101-107. doi:10.1016/j. lindif.2012.07.016.

Siegler, R., \& Araya, R. (2005). A computational model of conscious and unconscious strategy discovery. In Advances in child development and behavior (Vol. 33, pp. 1-42). Amsterdam: Elsevier. doi:10.1016/S0065-2407(05)80003-5.

St Clair-Thompson, H., \& Gathercole, S. E. (2006). Executive functions and achievement in school: Shifting, updating, inhibition, and working memory. The Quarterly Journal of Experimental Psychology, 59, 745-759. doi:10.1080/17470210500162854.

Swanson, H. L. (2011). Working memory, attention, and mathematical problem solving: A longitudinal study of elementary school children. Journal of Educational Psychology, 103(4), 821-837. doi:10.1037/a0025114.

Szucs, D., Devine, A., Soltez, F., Nobes, A., \& Gabriel, F. (2013). Developmental dyscalculia is related to visuo-spatial memory and inhibition impairment. Cortex, 49, 2674-2688. doi:10.1016/j. cortex.2013.06.007.

Torbeyns, J., Verschaffel, L., \& Ghesquière, P. (2006). The Development of children's adaptive expertise in the number domain 20 to 100. Cognition and Instruction, 24, 439-465. doi:10.1207/ s1532690xci2404_2.

Van der Ven, S. H. G., Kroesbergen, E. H., Boom, J., \& Leseman, P. P. M. (2012). The development of executive functions and early mathematics: A dynamic relationship. British Journal of Educational Psychology, 82, 100-119. doi:10.1111/j.2044-8279.2011.02035.x.

Visu-Petra, L., Cheie, L., Benga, O., \& Miclea, M. (2011). Cognitive control goes to school: The impact of executive functions on academic performance. Procedia Social and Behavioural Sciences, 11, 240-244. doi:10.1016/j.sbspro.2011.01.069.

Vorderman, C., Porkess, R., Budd, C., Dunne, R., \& Rahman-Hart, P. (2011). A world-class mathematics education for all our young people. http://www.tsm-resources.com/pdf/VordermanMathsReport.pdf. Accessed 5 May 2014.

Waber, D. P., Gerber, E. B., Turcios, V. Y., Wagner, E. R., \& Forbes, P. W. (2006). Executive functions and performance on high-stakes testing in children from urban schools. Developmental Neuropsychology, 29, 459-477. doi:10.1207/s15326942dn2903_5.

Wang, L. C., Tasi, H. J., \& Yang, H. M. (2012). Cognitive inhibition with and without dyslexia and dyscalculia. Research in Developmental Disabilities, 33, 1453-1461. doi:10.1207/ s15326942dn2903_5.

Wechsler, D. (2005). Wechsler individual achievement test. London: Harcourt Assessment.

Winegar, K.L. (2013). Inhibition performance in children with math disabilities. UC Riverside: Education. http://escholarship.org/uc/ item/2qx7h3h4 Accessed 5 May 2014.

Zhang, H. Y., \& Wu, H. R. (2011). Inhibitory ability of children with developmental dyscalculia. Journal of Huazhong University of Science and Technology-Medical Sciences, 31, 131-136. doi:10.1007/s11596-011-0164-2. 